\title{
Existence and Nonexistence of Traveling Waves for a Nonlocal Monostable Equation
}

\author{
By
}

Hiroki YAGISITA*

\begin{abstract}
We consider the nonlocal analogue of the Fisher-KPP equation

$$
u_{t}=\mu * u-u+f(u),
$$

where $\mu$ is a Borel-measure on $\mathbb{R}$ with $\mu(\mathbb{R})=1$ and $f$ satisfies $f(0)=f(1)=0$ and $f>0$ in $(0,1)$. We do not assume that $\mu$ is absolutely continuous with respect to the Lebesgue measure. The equation may have a standing wave solution whose profile is a monotone but discontinuous function. We show that there is a constant $c_{*}$ such that it has a traveling wave solution with speed $c$ when $c \geq c_{*}$ while no traveling wave solution with speed $c$ when $c<c_{*}$, provided $\int_{y \in \mathbb{R}} e^{-\lambda y} d \mu(y)<+\infty$ for some positive constant $\lambda$. In order to prove it, we modify a recursive method for abstract monotone discrete dynamical systems by Weinberger. We note that the monotone semiflow generated by the equation is not compact with respect to the compact-open topology. We also show that it has no traveling wave solution, provided $f^{\prime}(0)>0$ and $\int_{y \in \mathbb{R}} e^{-\lambda y} d \mu(y)=+\infty$ for all positive constants $\lambda$.
\end{abstract}

\section{$\S 1$. Introduction}

In 1930, Fisher [8] introduced the reaction-diffusion equation $u_{t}=u_{x x}+$ $u(1-u)$ as a model for the spread of an advantageous form (allele) of a single gene in a population of diploid individuals. He [9] found that there is a constant $c_{*}$ such that the equation has a traveling wave solution with speed $c$ when $c \geq c_{*}$

Communicated by H. Okamoto. Received November 1, 2008. Revised February 28, 2009. 2000 Mathematics Subject Classification(s): 35K57, 35K65, 35K90, 45 J05.

Key words: spreading speed, convolution model, integro-differential equation, discrete monostable equation, nonlocal evolution equation, Fisher-Kolmogorov equation.

* Department of Mathematics, Faculty of Science, Kyoto Sangyo University Motoyama, Kamigamo, Kita-Ku, Kyoto-City, 603-8555, Japan.

e-mail: hrk0ygst@cc.kyoto-su.ac.jp 
while it has no such solution when $c<c_{*}$. Kolmogorov, Petrovsky and Piskunov [16] obtained the same conclusion for a monostable equation $u_{t}=u_{x x}+f(u)$ with a more general nonlinearity $f$, and investigated long-time behavior in the model. Since the pioneering works, there have been extensive studies on traveling waves and long-time behavior for monostable evolution systems.

In this paper, we consider the following nonlocal analogue of the FisherKPP equation:

$$
u_{t}=\mu * u-u+f(u) .
$$

Here, $\mu$ is a Borel-measure on $\mathbb{R}$ with $\mu(\mathbb{R})=1$ and the convolution is defined by

$$
(\mu * u)(x):=\int_{y \in \mathbb{R}} u(x-y) d \mu(y)
$$

for a bounded and Borel-measurable function $u$ on $\mathbb{R}$. The nonlinearity $f$ is a Lipschitz continuous function on $\mathbb{R}$ with $f(0)=f(1)=0$ and $f>0$ in $(0,1)$. Then, $G(u):=\mu * u-u+f(u)$ is a map from the Banach space $L^{\infty}(\mathbb{R})$ into $L^{\infty}(\mathbb{R})$ and it is Lipschitz continuous. (We note that $u(x-y)$ is a Borel-measurable function on $\mathbb{R}^{2}$, and $\|u\|_{L^{\infty}(\mathbb{R})}=0$ implies $\|\mu * u\|_{L^{1}(\mathbb{R})} \leq$ $\int_{y \in \mathbb{R}}\left(\int_{x \in \mathbb{R}}|u(x-y)| d x\right) d \mu(y)=0$.) So, because the standard theory of ordinary differential equations works, we have well-posedness of the equation (1.1) and it generates a flow in $L^{\infty}(\mathbb{R})$.

For the nonlocal monostable equation, Atkinson and Reuter [1] first studied existence and nonexistence of traveling wave solutions. Schumacher [21, 22] showed that there is the minimal speed $c_{*}$ of traveling wave solutions and it has a traveling wave solution with speed $c$ when $c \geq c_{*}$, provided the extra condition $f(u) \leq f^{\prime}(0) u$ and some little ones. Here, we say that the solution $u(t, x)$ is $a$ traveling wave solution with profile $\psi$ and speed $c$, if $u(t, x) \equiv \psi\left(x-x_{0}+c t\right)$ holds for some constant $x_{0}$ with $0 \leq \psi \leq 1, \psi(-\infty)=0$ and $\psi(+\infty)=1$. Further, Coville, Dávila and Martínez [6] proved the following theorem:

Theorem ([6]). $\quad$ Suppose the nonlinearity $f \in C^{1}(\mathbb{R})$ satisfies $f^{\prime}(1)<0$ and the Borel-measure $\mu$ has a density function $J \in C(\mathbb{R})$ with

$$
\int_{y \in \mathbb{R}}\left(|y|+e^{-\lambda y}\right) J(y) d y<+\infty
$$

for some positive constant $\lambda$. Then, there exists a constant $c_{*}$ such that the equation (1.1) has a traveling wave solution with monotone profile and speed $c$ when $c \geq c_{*}$ while it has no such solution when $c<c_{*}$. 
Recently, the author [29] also obtained the following:

Theorem ([29]). Suppose there exists a positive constant $\lambda$ such that

$$
\int_{y \in \mathbb{R}} e^{\lambda|y|} d \mu(y)<+\infty
$$

holds. Then, there exists a constant $c_{*}$ such that the equation (1.1) has a traveling wave solution with monotone profile and speed $c$ when $c \geq c_{*}$ while it has no periodic traveling wave solution with average speed $c$ when $c<c_{*}$.

Here, a solution $\{u(t, x)\}_{t \in \mathbb{R}} \subset L^{\infty}(\mathbb{R})$ to $(1.1)$ is said to be a periodic traveling wave solution with average speed $c$, if there exists a positive constant $\tau$ such that $u(t+\tau, x)=u(t, x+c \tau)$ holds for all $t$ and $x \in \mathbb{R}$ with $0 \leq u(t, x) \leq 1$, $\lim _{x \rightarrow+\infty} u(t, x)=1$ and $\|u(t, x)-1\|_{L^{\infty}(\mathbb{R})} \neq 0$.

The goal of this paper is to improve this result of [29], and the following two theorems are the main results:

Theorem 1. Suppose there exists a positive constant $\lambda$ such that

$$
\int_{y \in \mathbb{R}} e^{-\lambda y} d \mu(y)<+\infty
$$

holds. Then, there exists a constant $c_{*}$ such that the equation (1.1) has a traveling wave solution with monotone profile and speed $c$ when $c \geq c_{*}$ while it has no periodic traveling wave solution with average speed $c$ when $c<c_{*}$.

Here, a solution $\{u(t, x)\}_{t \in \mathbb{R}} \subset L^{\infty}(\mathbb{R})$ to $(1.1)$ is said to be a traveling wave solution with monotone profile and speed $c$, if there exists a monotone nondecreasing function $\psi$ on $\mathbb{R}$ with $\psi(-\infty)=0$ and $\psi(+\infty)=1$ such that $u(t, x) \equiv \psi(x+c t)$ holds. Also, a solution $\{u(t, x)\}_{t \in \mathbb{R}} \subset L^{\infty}(\mathbb{R})$ to (1.1) is said to be a periodic traveling wave solution with average speed $c$, if there exists a positive constant $\tau$ such that $u(t+\tau, x)=u(t, x+c \tau)$ holds for all $t$ and $x \in \mathbb{R}$ with $0 \leq u(t, x) \leq 1, \lim _{x \rightarrow+\infty} u(t, x)=1$ and $\|u(t, x)-1\|_{L^{\infty}(\mathbb{R})} \neq 0$.

Remark. If a solution is a traveling wave with speed $c$, then it is a periodic traveling wave with average speed $c$. The converse may not hold. In fact, if $\mu(\mathbb{Z})=1$ holds and there exists a positive constant $\lambda$ such that $\int_{y \in \mathbb{R}} e^{-\lambda y} d \mu(y)<+\infty$ holds, then there exists a solution $u$ to (1.1) such that $u$ is not a traveling wave but $u$ is a periodic traveling wave with monotone profile. Here, we represent the idea of the proof. By Theorem 1, there exist a positive constant $c$ and a sequence $\left\{u_{n}(t)\right\}_{n \in \mathbb{Z}} \subset C^{1}(\mathbb{R})$ such that

$$
\frac{d u_{n}}{d t}(t)=\left(\sum_{m \in \mathbb{Z}} \mu(\{m\}) u_{n-m}(t)\right)-u_{n}(t)+f\left(u_{n}(t)\right),
$$




$$
u_{n}(t) \leq u_{n+1}(t), \lim _{n \rightarrow-\infty} u_{n}(t)=0, \lim _{n \rightarrow+\infty} u_{n}(t)=1
$$

and

$$
u_{n}\left(t+\frac{1}{c}\right)=u_{n+1}(t)
$$

hold. Then, the function $u(t, x):=u_{[x]}(t)$ is a periodic traveling wave with the minimal period $\frac{1}{c}$, where [.] is Gauss' symbol.

Theorem 2. $\quad$ Suppose the measure $\mu$ satisfies

$$
\int_{y \in \mathbb{R}} e^{-\lambda y} d \mu(y)=+\infty
$$

for all positive constants $\lambda$. Suppose the nonlinearity $f \in C^{1}(\mathbb{R})$ satisfies

$$
f^{\prime}(0)>0
$$

Then, the equation (1.1) has no periodic traveling wave solution.

Remark. When $\int_{y \in \mathbb{R}} e^{-\lambda y} d \mu(y)=+\infty$ holds for all positive constants $\lambda$ and $f^{\prime}(0)=0$ holds, we do not know whether there exists a (periodic) traveling wave. In Section 5 below, we only see Theorem 19 and Lemma 20.

In these results, we do not assume that the measure $\mu$ is absolutely continuous with respect to the Lebesgue measure. For example, not only the integrodifferential equation

$$
\frac{\partial u}{\partial t}(t, x)=\int_{0}^{1} u(t, x-y) d y-u(t, x)+f(u(t, x))
$$

but also the discrete equation

$$
\frac{\partial u}{\partial t}(t, x)=u(t, x-1)-u(t, x)+f(u(t, x))
$$

satisfies the assumption of Theorem 1 for the measure $\mu$. In order to prove these results, we employ the recursive method for monotone dynamical systems by Weinberger [25] and Li, Weinberger and Lewis [17]. We note that the semiflow generated by the equation (1.1) is not compact with respect to the compactopen topology. See Propositions 16 and 17 of [29] for a simple condition for the profile of a standing wave solution (i.e., a traveling wave solution with speed $0)$ to be a discontinuous function.

Schumacher [21, 22], Carr and Chmaj [3] and Coville, Dávila and Martínez [6] also studied uniqueness of traveling wave solutions. In [6], we could see an 
interesting example of nonuniqueness, where the equation (1.1) admits infinitely many monotone profiles for standing wave solutions but it admits no continuous one. See, e.g., $[5,7,10,11,12,13,14,15,18,19,23,24,26,27,28]$ on traveling waves and long-time behavior in various monostable evolution systems, [2, 4, 30] nonlocal bistable equations and [20] Euler equation.

In Section 2, we recall abstract results for monotone semiflows from [29]. The results give abstract conditions such that a semiflow satisfying the conditions has a traveling wave solution with speed $c$ when $c \geq c_{*}$ while it has no periodic traveling wave solution with average speed $c$ when $c<c_{*}$. In Section 3 , we also repeat the proof given in [29] for reader's convenience. In Section 4 , we give basic facts for nonlocal equations in $L^{\infty}(\mathbb{R})$. In Section 5 , we prove Theorem 1. In Section 6, we recall a result on spreading speeds by Weinberger [25]. In Section 7, we prove Theorem 2. In a sequel [30] to this paper, the author shall refer several results from this paper.

\section{§2. Abstract Results for Monotone Semiflows}

In this section, we recall some abstract results for monotone semiflows from [29]. (In Section 3 below, we would prove them for reader's convenience.) Put a set of functions on $\mathbb{R}$;

$$
\mathcal{M}:=\{u \mid u \text { is a monotone nondecreasing }
$$

and left continuous function on $\mathbb{R}$ with $0 \leq u \leq 1\}$.

The followings are basic conditions for discrete dynamical systems on $\mathcal{M}$ :

Hypotheses 3. Let $Q_{0}$ be a map from $\mathcal{M}$ into $\mathcal{M}$.

(i) $Q_{0}$ is continuous in the following sense: If a sequence $\left\{u_{k}\right\}_{k \in \mathbb{N}} \subset \mathcal{M}$ converges to $u \in \mathcal{M}$ uniformly on every bounded interval, then the sequence $\left\{Q_{0}\left[u_{k}\right]\right\}_{k \in \mathbb{N}}$ converges to $Q_{0}[u]$ almost everywhere.

(ii) $Q_{0}$ is order preserving; i.e.,

$$
u_{1} \leq u_{2} \Longrightarrow Q_{0}\left[u_{1}\right] \leq Q_{0}\left[u_{2}\right]
$$

for all $u_{1}$ and $u_{2} \in \mathcal{M}$. Here, $u \leq v$ means that $u(x) \leq v(x)$ holds for all $x \in \mathbb{R}$.

(iii) $Q_{0}$ is translation invariant; i.e.,

$$
T_{x_{0}} Q_{0}=Q_{0} T_{x_{0}}
$$

for all $x_{0} \in \mathbb{R}$. Here, $T_{x_{0}}$ is the translation operator defined by $\left(T_{x_{0}}[u]\right)(\cdot):=$ $u\left(\cdot-x_{0}\right)$. 
(iv) $Q_{0}$ is monostable; i.e.,

$$
0<\gamma<1 \Longrightarrow \gamma<Q_{0}[\gamma]
$$

for all constant functions $\gamma$.

Remark. $\quad 1^{\circ}$. If $Q_{0}$ satisfies Hypothesis 3 (iii), then $Q_{0}$ maps constant functions to constant functions. $2^{\circ}$. The semiflow generated by a map $Q_{0}$ satisfying Hypotheses 3 may not be compact with respect to the compact-open topology.

We add the following conditions to Hypotheses 3 for continuous dynamical systems on $\mathcal{M}$ :

Hypotheses 4. Let $Q:=\left\{Q^{t}\right\}_{t \in[0,+\infty)}$ be a family of maps from $\mathcal{M}$ to $\mathcal{M}$.

(i) $Q$ is a semigroup; i.e., $Q^{t} \circ Q^{s}=Q^{t+s}$ for all $t$ and $s \in[0,+\infty)$.

(ii) $Q$ is continuous in the following sense: Suppose a sequence $\left\{t_{k}\right\}_{k \in \mathbb{N}} \subset$ $[0,+\infty)$ converges to 0 , and $u \in \mathcal{M}$. Then, the sequence $\left\{Q^{t_{k}}[u]\right\}_{k \in \mathbb{N}}$ converges to $u$ almost everywhere.

From [29], we recall the following two results for continuous dynamical systems on $\mathcal{M}$ :

Theorem 5. Let $Q^{t}$ be a map from $\mathcal{M}$ to $\mathcal{M}$ for $t \in[0,+\infty)$. Suppose $Q^{t}$ satisfies Hypotheses 3 for all $t \in(0,+\infty)$, and $Q:=\left\{Q^{t}\right\}_{t \in[0,+\infty)}$ Hypotheses 4. Then, the following holds:

Let $c \in \mathbb{R}$. Suppose there exist $\tau \in(0,+\infty)$ and $\phi \in \mathcal{M}$ with $\left(Q^{\tau}[\phi]\right)(x-$ $c \tau) \leq \phi(x), \phi \not \equiv 0$ and $\phi \not \equiv 1$. Then, there exists $\psi \in \mathcal{M}$ with $\psi(-\infty)=0$ and $\psi(+\infty)=1$ such that $\left(Q^{t}[\psi]\right)(x-c t) \equiv \psi(x)$ holds for all $t \in[0,+\infty)$.

Theorem 6. Let $Q^{t}$ be a map from $\mathcal{M}$ to $\mathcal{M}$ for $t \in[0,+\infty)$. Suppose $Q^{t}$ satisfies Hypotheses 3 for all $t \in(0,+\infty)$, and $Q:=\left\{Q^{t}\right\}_{t \in[0,+\infty)}$ Hypotheses 4 . Then, there exists $c_{*} \in(-\infty,+\infty]$ such that the following holds:

Let $c \in \mathbb{R}$. Then, there exists $\psi \in \mathcal{M}$ with $\psi(-\infty)=0$ and $\psi(+\infty)=1$ such that $\left(Q^{t}[\psi]\right)(x-c t) \equiv \psi(x)$ holds for all $t \in[0,+\infty)$ if and only if $c \geq c_{*}$.

Remark. For Theorem 6, we note that there do not exist a constant $c$ and $\psi \in \mathcal{M}$ with $\psi(-\infty)=0$ and $\psi(+\infty)=1$ such that $\left(Q^{t}[\psi]\right)(x-c t) \equiv \psi(x)$ holds for all $t \in[0,+\infty)$ if and only if $c_{*}=+\infty$. 


\section{§3. Proof of the Abstract Theorems}

In Section 3 of [29], the author already proved Theorems 5 and 6 . He modified the recursive method introduced by Weinberger [25] and Li, Weinberger and Lewis [17]. In this section, we would repeat the proof for reader's convenience. Some results stated in this section would be also useful to a sequel [30].

Lemma 7. Let a sequence $\left\{u_{k}\right\}_{k \in \mathbb{N}}$ of monotone nondecreasing functions on $\mathbb{R}$ converge to a continuous function $u$ on $\mathbb{R}$ almost everywhere. Then, $\left\{u_{k}\right\}_{k \in \mathbb{N}}$ converges to u uniformly on every bounded interval.

Proof. Let $C \in(0,+\infty)$ and $\varepsilon \in(0,+\infty)$. Then, there exists $\delta \in(0,+\infty)$ such that, for any $y_{1}$ and $y_{2} \in[-C-1,+C+1],\left|y_{2}-y_{1}\right|<\delta$ implies $\left|u\left(y_{2}\right)-u\left(y_{1}\right)\right|<\varepsilon / 4$. We take $N \in \mathbb{N}$ and a sequence $\left\{x_{n}\right\}_{n=1}^{N}$ such that $\lim _{k \rightarrow \infty} u_{k}\left(x_{n}\right)=u\left(x_{n}\right),-C-1 \leq x_{1} \leq-C, x_{n}<x_{n+1}<x_{n}+\delta$ and $+C \leq x_{N} \leq+C+1$ hold.

Let $k \in \mathbb{N}$ be sufficiently large. Then, $\max \left\{\left|u_{k}\left(x_{n}\right)-u\left(x_{n}\right)\right|\right\}_{n=1}^{N}<\varepsilon / 4$ holds. Let $x \in[-C,+C]$. There exists $n$ such that $x_{n} \leq x \leq x_{n+1}$ holds. So, we get $\left|u_{k}(x)-u(x)\right| \leq\left|u_{k}\left(x_{n}\right)-u(x)\right|+\left|u_{k}\left(x_{n+1}\right)-u(x)\right| \leq\left|u_{k}\left(x_{n}\right)-u\left(x_{n}\right)\right|+$ $\left|u\left(x_{n}\right)-u(x)\right|+\left|u_{k}\left(x_{n+1}\right)-u\left(x_{n+1}\right)\right|+\left|u\left(x_{n+1}\right)-u(x)\right|<\varepsilon$.

The set of discontinuous points of a monotone function on $\mathbb{R}$ is at most countable. So, if a sequence $\left\{u_{k}\right\}_{k \in \mathbb{N}}$ of monotone functions on $\mathbb{R}$ converges to a monotone function $u$ on $\mathbb{R}$ at every continuous point of $u$, then it converges to $u$ almost everywhere. The converse also holds:

Lemma 8. Let a sequence $\left\{u_{k}\right\}_{k \in \mathbb{N}}$ of monotone nondecreasing functions on $\mathbb{R}$ converge to a monotone nondecreasing function $u$ on $\mathbb{R}$ almost everywhere. Then, $\lim _{k \rightarrow \infty} u_{k}(x)=u(x)$ holds for all continuous points $x \in \mathbb{R}$ of $u$.

Proof. For $n \in \mathbb{N}$, we take $\underline{x}_{n} \in\left(x-2^{-n}, x\right]$ and $\bar{x}_{n} \in\left[x, x+2^{-n}\right)$ satisfying $\lim _{k \rightarrow \infty} u_{k}\left(\underline{x}_{n}\right)=u\left(\underline{x}_{n}\right)$ and $\lim _{k \rightarrow \infty} u_{k}\left(\bar{x}_{n}\right)=u\left(\bar{x}_{n}\right)$. Then, $u\left(\underline{x}_{n}\right) \leq$ $\liminf \operatorname{in}_{k \rightarrow \infty} u_{k}(x) \leq \lim \sup _{k \rightarrow \infty} u_{k}(x) \leq u\left(\bar{x}_{n}\right)$ holds. Hence, we have $\lim _{k \rightarrow \infty}$ $u_{k}(x)=u(x)$ as $x$ is a continuous point of $u$.

Hypotheses 3 imply more strong continuity than Hypothesis 3 (i): 
Proposition 9. Let a map $Q_{0}: \mathcal{M} \rightarrow \mathcal{M}$ satisfy Hypotheses 3 (i), (ii) and (iii). Suppose a sequence $\left\{u_{k}\right\}_{k \in \mathbb{N}} \subset \mathcal{M}$ converges to $u \in \mathcal{M}$ almost everywhere. Then, $\lim _{k \rightarrow \infty}\left(Q_{0}\left[u_{k}\right]\right)(x)=\left(Q_{0}[u]\right)(x)$ holds for all continuous points $x \in \mathbb{R}$ of $Q_{0}[u]$.

Proof. We take a cutoff function $\rho \in C^{\infty}(\mathbb{R})$ with

$$
\begin{aligned}
& |x| \geq 1 / 2 \Longrightarrow \rho(x)=0, \\
& |x|<1 / 2 \Longrightarrow \rho(x)>0
\end{aligned}
$$

and

$$
\int_{x \in \mathbb{R}} \rho(x) d x=1 .
$$

For $n \in \mathbb{N}$, we put smooth functions

$$
\begin{gathered}
\rho_{n}(\cdot):=2^{n} \rho\left(2^{n} \cdot\right), \\
\underline{u}^{n}(\cdot):=\left(\rho_{n} * u\right)\left(\cdot-2^{-(n+1)}\right)
\end{gathered}
$$

and

$$
\bar{u}^{n}(\cdot):=\left(\rho_{n} * u\right)\left(\cdot+2^{-(n+1)}\right)
$$

Then, we obtain

$$
u\left(\cdot-2^{-n}\right) \leq \underline{u}^{n}(\cdot) \leq u(\cdot) \leq \bar{u}^{n}(\cdot) \leq u\left(\cdot+2^{-n}\right) .
$$

The sequence $\left\{\min \left\{u_{k}, \underline{u}^{n}\right\}\right\}_{k \in \mathbb{N}}$ converges to $\underline{u}^{n}$ almost everywhere, and $\left\{\max \left\{u_{k}, \bar{u}^{n}\right\}\right\}_{k \in \mathbb{N}}$ also $\bar{u}^{n}$. Hence, by Lemma 7 , the sequence $\left\{\min \left\{u_{k}, \underline{u}^{n}\right\}\right\}_{k \in \mathbb{N}}$ converges to $\underline{u}^{n}$ uniformly on every bounded interval, and $\left\{\max \left\{u_{k}, \bar{u}^{n}\right\}\right\}_{k \in \mathbb{N}}$ also $\bar{u}^{n}$. Then, by Hypothesis $3(\mathrm{i})$, the sequence $\left\{Q_{0}\left[\min \left\{u_{k}, \underline{u}^{n}\right\}\right]\right\}_{k \in \mathbb{N}}$ converges to $Q_{0}\left[\underline{u}^{n}\right]$ almost everywhere, and $\left\{Q_{0}\left[\max \left\{u_{k}, \bar{u}^{n}\right\}\right]\right\}_{k \in \mathbb{N}}$ also $Q_{0}\left[\bar{u}^{n}\right]$. From Hypothesis 3 (ii), $Q_{0}\left[\min \left\{u_{k}, \underline{u}^{n}\right\}\right] \leq Q_{0}\left[u_{k}\right] \leq Q_{0}\left[\max \left\{u_{k}, \bar{u}^{n}\right\}\right]$ holds. Therefore, $Q_{0}\left[\underline{u}^{n}\right] \leq \liminf _{k \rightarrow \infty} Q_{0}\left[u_{k}\right] \leq \lim \sup _{k \rightarrow \infty} Q_{0}\left[u_{k}\right] \leq Q_{0}\left[\bar{u}^{n}\right]$ holds almost everywhere. So, by Hypotheses 3 (ii) and (iii), $Q_{0}[u]\left(\cdot-2^{-n}\right) \leq$ $\liminf _{k \rightarrow \infty} Q_{0}\left[u_{k}\right](\cdot) \leq \limsup _{k \rightarrow \infty} Q_{0}\left[u_{k}\right](\cdot) \leq Q_{0}[u]\left(\cdot+2^{-n}\right)$ holds almost everywhere. Hence, $\lim _{k \rightarrow \infty} Q_{0}\left[u_{k}\right](\cdot)=Q_{0}[u](\cdot)$ holds almost everywhere, because $\lim _{n \rightarrow \infty} Q_{0}[u]\left(\cdot-2^{-n}\right)=\lim _{n \rightarrow \infty} Q_{0}[u]\left(\cdot+2^{-n}\right)=Q_{0}[u](\cdot)$ holds almost everywhere. So, from Lemma $8, \lim _{k \rightarrow \infty}\left(Q_{0}\left[u_{k}\right]\right)(x)=\left(Q_{0}[u]\right)(x)$ holds for all continuous points $x \in \mathbb{R}$ of $Q_{0}[u]$. 
Combining Proposition 9 with Helly's theorem, we can make the argument of Weinberger [25] and Li, Weinberger and Lewis [17] work to obtain the following proposition. It states that existence of suitable super-solutions of the form $\left\{v_{n}(x+c n)\right\}_{n=0}^{\infty}$ implies existence of traveling wave solutions with speed $c$ in the discrete dynamical systems on $\mathcal{M}$ :

Proposition 10. Let a map $Q_{0}: \mathcal{M} \rightarrow \mathcal{M}$ satisfy Hypotheses 3, and $c \in \mathbb{R}$. Suppose there exists a sequence $\left\{v_{n}\right\}_{n=0}^{\infty} \subset \mathcal{M}$ with $\left(Q_{0}\left[v_{n}\right]\right)(x-c) \leq$ $v_{n+1}(x), \inf _{n=0,1,2, \cdots} v_{n}(x) \not \equiv 0$ and $\liminf _{n \rightarrow \infty} v_{n}(x) \not \equiv 1$. Then, there exists $\psi \in \mathcal{M}$ with $\left(Q_{0}[\psi]\right)(x-c) \equiv \psi(x), \psi(-\infty)=0$ and $\psi(+\infty)=1$.

Proof. We put $w(\cdot):=\lim _{h \downarrow+0} \inf _{n=0,1,2, \cdots} v_{n}(\cdot-h)$, and $u_{0}^{k}:=2^{-k} w \in$ $\mathcal{M}$ for $k \in \mathbb{N}$. We also take functions $u_{n}^{k} \in \mathcal{M}$ such that

$$
u_{n}^{k}(\cdot)=\max \left\{Q_{0}\left[u_{n-1}^{k}\right](\cdot-c), 2^{-k} w(\cdot)\right\}
$$

holds for $k$ and $n \in \mathbb{N}$.

We show $u_{n}^{k} \leq u_{n+1}^{k}$. We have $u_{0}^{k} \leq u_{1}^{k}$. As $u_{n-1}^{k} \leq u_{n}^{k}$ holds, we get $Q_{0}\left[u_{n-1}^{k}\right] \leq Q_{0}\left[u_{n}^{k}\right]$ and $u_{n}^{k} \leq u_{n+1}^{k}$. So, we have

$$
u_{n}^{k} \leq u_{n+1}^{k} .
$$

In virtue of (3.2), we put $u^{k}:=\lim _{n \rightarrow \infty} u_{n}^{k} \in \mathcal{M}$. Then, by (3.1) and Proposition 9 ,

$$
u^{k}(\cdot)=\max \left\{Q_{0}\left[u^{k}\right](\cdot-c), 2^{-k} w(\cdot)\right\}
$$

holds. Because $\lim _{m \rightarrow \infty} Q_{0}\left[u^{k}(\cdot+m)\right]=Q_{0}\left[u^{k}(+\infty)\right]$ holds from Proposition 9 , we have

$$
\begin{gathered}
u^{k}(+\infty)=\lim _{m \rightarrow \infty} \max \left\{Q_{0}\left[u^{k}\right](m-c), 2^{-k} w(m)\right\} \\
=\lim _{m \rightarrow \infty} \max \left\{Q_{0}\left[u^{k}(\cdot+m)\right](-c), 2^{-k} w(m)\right\} \\
=\max \left\{Q_{0}\left[u^{k}(+\infty)\right], 2^{-k} w(+\infty)\right\} .
\end{gathered}
$$

Hence, $u^{k}(+\infty) \geq Q_{0}\left[u^{k}(+\infty)\right]$ and $u^{k}(+\infty) \geq 2^{-k} w(+\infty)>0$ hold. So, because $\left\{\gamma \in \mathbb{R} \mid 0 \leq \gamma \leq 1, \gamma \geq Q_{0}[\gamma]\right\} \subset\{0,1\}$ holds from Hypothesis 3 (iv), we obtain

$$
u^{k}(+\infty)=1 .
$$

We show $u_{n}^{k} \leq v_{n}$. We get $u_{0}^{k} \leq w \leq v_{0}$. As $u_{n-1}^{k} \leq v_{n-1}$ holds, we have

$$
Q_{0}\left[u_{n-1}^{k}\right](\cdot-c) \leq Q_{0}\left[v_{n-1}\right](\cdot-c) \leq v_{n}(\cdot)
$$


and $u_{n}^{k} \leq v_{n}$ because of $2^{-k} w \leq w \leq v_{n}$. So, we have

$$
u_{n}^{k} \leq v_{n} .
$$

From (3.5), we see

$$
u^{k}(-\infty) \leq \lim _{m \rightarrow \infty} \liminf _{n \rightarrow \infty} v_{n}(-m)<1 .
$$

Also, $\lim _{m \rightarrow \infty} Q_{0}\left[u^{k}(\cdot-m)\right]=Q_{0}\left[u^{k}(-\infty)\right]$ holds from Proposition 9. Hence, by (3.3), we have

$$
u^{k}(-\infty)=\lim _{m \rightarrow \infty} \max \left\{Q_{0}\left[u^{k}\right](-m-c), 2^{-k} w(-m)\right\} \geq Q_{0}\left[u^{k}(-\infty)\right] .
$$

So, from Hypothesis 3 (iv) and (3.6), we obtain

$$
u^{k}(-\infty)=0 .
$$

In virtue of (3.4) and (3.7), there exists $x_{k}$ such that $u^{k}\left(-x_{k}\right) \leq 1 / 2 \leq$ $\lim _{h \downarrow+0} u^{k}\left(-x_{k}+h\right)$ for $k \in \mathbb{N}$. We put $\psi^{k}(\cdot):=u^{k}\left(\cdot-x_{k}\right) \in \mathcal{M}$. Then, we have

$$
\psi^{k}(0) \leq 1 / 2 \leq \lim _{h \downarrow+0} \psi^{k}(h)
$$

and

$$
\psi^{k}(\cdot)=\max \left\{Q_{0}\left[\psi^{k}\right](\cdot-c), 2^{-k} w\left(\cdot-x_{k}\right)\right\}
$$

from (3.3). By Helly's theorem, there exist a subsequence $\{k(n)\}_{n \in \mathbb{N}}$ and $\psi \in$ $\mathcal{M}$ such that $\lim _{n \rightarrow \infty} \psi^{k(n)}(x)=\psi(x)$ holds for all continuous points $x \in \mathbb{R}$ of $\psi$. So, from (3.8), (3.9) and Proposition 9,

$$
\psi(0) \leq 1 / 2 \leq \lim _{h \downarrow+0} \psi(h)
$$

and

$$
\psi(\cdot)=Q_{0}[\psi](\cdot-c)
$$

holds. Because $\psi(-\infty)=Q_{0}[\psi(-\infty)]$ and $\psi(+\infty)=Q_{0}[\psi(+\infty)]$ also hold by (3.11) and Proposition 9, from Hypothesis 3 (iv) and (3.10), we have $\psi(-\infty)=$ 0 and $\psi(+\infty)=1$.

In the discrete dynamical system on $\mathcal{M}$ generated by a map $Q_{0}$ satisfying Hypotheses 3, if there is a periodic traveling wave super-solution with average speed $c$, then there is a traveling wave solution with speed $c$ : 
Theorem 11. Let a map $Q_{0}: \mathcal{M} \rightarrow \mathcal{M}$ satisfy Hypotheses 3, and $c \in \mathbb{R}$. Suppose there exist $\tau \in \mathbb{N}$ and $\phi \in \mathcal{M}$ with $\left(Q_{0}{ }^{\tau}[\phi]\right)(x-c \tau) \leq \phi(x)$, $\phi \not \equiv 0$ and $\phi \not \equiv 1$. Then, there exists $\psi \in \mathcal{M}$ with $\left(Q_{0}[\psi]\right)(x-c) \equiv \psi(x)$, $\psi(-\infty)=0$ and $\psi(+\infty)=1$.

Proof. We take functions $v_{n} \in \mathcal{M}$ for $n=0,1,2, \cdots$ such that

$$
v_{n+m \tau}=\left(Q_{0}{ }^{n}[\phi]\right)(\cdot-c n)
$$

holds for all $n=0,1,2, \cdots, \tau-1$ and $m=0,1,2, \cdots$. Then, we see

$$
v_{n+1}(\cdot) \geq Q_{0}\left[v_{n}\right](\cdot-c)
$$

and

$$
\liminf _{n \rightarrow \infty} v_{n}=\inf _{n=0,1,2, \cdots} v_{n}=\min _{n=0,1,2, \cdots, \tau-1} v_{n} .
$$

We show $v_{n}(+\infty)>0$. We have $v_{0}(+\infty)>0$. As $v_{n-1}(+\infty)>0$ holds, we get $v_{n}(+\infty) \geq Q_{0}\left[v_{n-1}(+\infty)\right]>0$ by (3.12), Proposition 9 , Hypotheses 3 (ii) and (iv). So, we have $v_{n}(+\infty)>0$. Hence, because $\lim _{m \rightarrow \infty} \min _{n=0,1,2, \cdots, \tau-1} v_{n}$ $(m)>0$ holds, from (3.13), we see $\inf _{n=0,1,2, \cdots} v_{n} \not \equiv 0$. Because $\min _{n=0,1,2, \cdots, \tau-1}$ $v_{n} \leq \phi$ holds, by (3.13) and $\phi(-\infty)<1$, we have $\liminf _{n \rightarrow \infty} v_{n} \not \equiv 1$. Therefore, by Proposition 10, there exists $\psi \in \mathcal{M}$ with $Q_{0}[\psi](\cdot-c)=\psi(\cdot), \psi(-\infty)=0$ and $\psi(+\infty)=1$.

Lemma 12. Let a sequence $\left\{u_{k}\right\}_{k \in \mathbb{N}}$ of monotone nondecreasing functions on $\mathbb{R}$ converge to a monotone nondecreasing function $u$ on $\mathbb{R}$ almost everywhere. Then, $\lim _{k \rightarrow \infty} u_{k}\left(x_{k}\right)=u(x)$ holds for all continuous points $x \in \mathbb{R}$ of $u$ and sequences $\left\{x_{k}\right\}_{k \in \mathbb{N}} \subset \mathbb{R}$ with $\lim _{k \rightarrow \infty} x_{k}=x$.

Proof. We put $h_{n}:=\sup _{k=n, n+1, n+2, \ldots}\left|x_{k}-x\right|$ for $n \in \mathbb{N}$. Then, $u_{k}(\cdot-$ $\left.h_{n}\right) \leq u_{k}\left(\cdot+\left(x_{k}-x\right)\right) \leq u_{k}\left(\cdot+h_{n}\right)$ holds when $k \geq n$. Hence, $u\left(\cdot-h_{n}\right) \leq$ $\liminf _{k \rightarrow \infty} u_{k}\left(\cdot+\left(x_{k}-x\right)\right) \leq \limsup _{k \rightarrow \infty} u_{k}\left(\cdot+\left(x_{k}-x\right)\right) \leq u\left(\cdot+h_{n}\right)$ holds almost everywhere. So, $\lim _{k \rightarrow \infty} u_{k}\left(\cdot+\left(x_{k}-x\right)\right)=u(\cdot)$ holds almost everywhere, because $\lim _{n \rightarrow \infty} u\left(\cdot-h_{n}\right)=\lim _{n \rightarrow \infty} u\left(\cdot+h_{n}\right)=u(\cdot)$ holds almost everywhere. Hence, from Lemma $8, \lim _{k \rightarrow \infty} u_{k}\left(x_{k}\right)=\lim _{k \rightarrow \infty} u_{k}\left(x+\left(x_{k}-x\right)\right)=u(x)$ holds.

The infimum $c_{*}$ of the speeds of traveling wave solutions is not $-\infty$, and there is a traveling wave solution with speed $c$ when $c \geq c_{*}$ : 
Theorem 13. $\quad$ Suppose a map $Q_{0}: \mathcal{M} \rightarrow \mathcal{M}$ satisfies Hypotheses 3. Then, there exists $c_{*} \in(-\infty,+\infty]$ such that the following holds:

Let $c \in \mathbb{R}$. Then, there exists $\psi \in \mathcal{M}$ with $\left(Q_{0}[\psi]\right)(x-c \tau) \equiv \psi(x)$, $\psi(-\infty)=0$ and $\psi(+\infty)=1$ if and only if $c \geq c_{*}$.

Proof. [Step 1] Let $c_{*} \in[-\infty,+\infty]$ denote the infimum of $c \in \mathbb{R}$ such that there exists $\psi \in \mathcal{M}$ with $Q_{0}[\psi](\cdot-c)=\psi(\cdot), \psi(-\infty)=0$ and $\psi(+\infty)=1$. Then, we have the following: Let $c \in \mathbb{R}$. Then, there exists $\psi \in \mathcal{M}$ with $Q_{0}[\psi](\cdot-c)=\psi(\cdot), \psi(-\infty)=0$ and $\psi(+\infty)=1$ only if $c \geq c_{*}$.

[Step 2] In this step, we show the following: If $c \in\left(c_{*},+\infty\right)$, then there exists $\psi \in \mathcal{M}$ with $Q_{0}[\psi](\cdot-c)=\psi(\cdot), \psi(-\infty)=0$ and $\psi(+\infty)=1$.

There exist $c_{0} \in(-\infty, c)$ and $\phi \in \mathcal{M}$ with $Q_{0}[\phi]\left(\cdot-c_{0}\right)=\phi(\cdot), \phi(-\infty)=0$ and $\phi(+\infty)=1$. Then, because we have $Q_{0}[\phi](\cdot-c) \leq \phi(\cdot)$, by Theorem 11, there exists $\psi \in \mathcal{M}$ with $Q_{0}[\psi](\cdot-c)=\psi(\cdot), \psi(-\infty)=0$ and $\psi(+\infty)=1$.

[Step 3] In this step, we show the following: If $c_{*} \in \mathbb{R}$, then there exists $\psi \in \mathcal{M}$ with $Q_{0}[\psi]\left(\cdot-c_{*}\right)=\psi(\cdot), \psi(-\infty)=0$ and $\psi(+\infty)=1$.

In virtue of Step 2 , there exists $\psi_{k} \in \mathcal{M}$ with $Q_{0}\left[\psi_{k}\right]\left(\cdot-\left(c_{*}+2^{-k}\right)\right)=$ $\psi_{k}(\cdot), \psi_{k}(-\infty)=0$ and $\psi_{k}(+\infty)=1$ for $k \in \mathbb{N}$. We also take $x_{k}$ such that $\psi_{k}\left(-x_{k}\right) \leq 1 / 2 \leq \lim _{h \downarrow+0} \psi_{k}\left(-x_{k}+h\right)$, and put $\psi^{k}(\cdot):=\psi_{k}\left(\cdot-x_{k}\right) \in \mathcal{M}$. Then, we have

$$
\psi^{k}(0) \leq 1 / 2 \leq \lim _{h \downarrow+0} \psi^{k}(h)
$$

and

$$
Q_{0}\left[\psi^{k}\left(\cdot-2^{-k}\right)\right]\left(\cdot-c_{*}\right)=\psi^{k}(\cdot) .
$$

By Helly's theorem, there exist a subsequence $\{k(n)\}_{n \in \mathbb{N}}$ and $\psi \in \mathcal{M}$ such that $\lim _{n \rightarrow \infty} \psi^{k(n)}(x)=\psi(x)$ holds for all continuous points $x \in \mathbb{R}$ of $\psi$. Also, by Lemma 12, $\lim _{n \rightarrow \infty} \psi^{k(n)}\left(x-2^{-k(n)}\right)=\psi(x)$ holds for all continuous points $x \in \mathbb{R}$ of $\psi$. Therefore, from (3.14), (3.15) and Proposition 9,

$$
\psi(0) \leq 1 / 2 \leq \lim _{h \downarrow+0} \psi(h)
$$

and

$$
Q_{0}[\psi]\left(\cdot-c_{*}\right)=\psi(\cdot)
$$

holds. Because $Q_{0}[\psi(-\infty)]=\psi(-\infty)$ and $Q_{0}[\psi(+\infty)]=\psi(+\infty)$ also hold by (3.17) and Proposition 9, from Hypothesis 3 (iv) and (3.16), we have $\psi(-\infty)=$ 0 and $\psi(+\infty)=1$. 
[Step 4] Finally, we show $c_{*} \in(-\infty,+\infty]$.

Suppose $c_{*}=-\infty$. Then, in virtue of Step 2, there exists $\phi_{k} \in \mathcal{M}$ with $Q_{0}\left[\phi_{k}\right]\left(\cdot+2^{k}\right)=\phi_{k}(\cdot), \phi_{k}(-\infty)=0$ and $\phi_{k}(+\infty)=1$ for $k \in \mathbb{N}$. We also take $x_{k}$ such that $\phi_{k}\left(-x_{k}\right) \leq 1 / 2 \leq \lim _{h \downarrow+0} \phi_{k}\left(-x_{k}+h\right)$, and put $\phi^{k}(\cdot):=$ $\phi_{k}\left(\cdot-x_{k}\right) \in \mathcal{M}$. Then, we have

$$
\phi^{k}(0) \leq 1 / 2 \leq \lim _{h \downarrow+0} \phi^{k}(h)
$$

and

$$
Q_{0}\left[\phi^{k}\left(\cdot+2^{k}\right)\right](\cdot)=\phi^{k}(\cdot) .
$$

Put $\chi \in \mathcal{M}$ such that $\chi(x)=0(x \leq 0)$ and $\chi(x)=1 / 2(0<x)$. Then, $\chi \leq \phi^{k}$ holds from (3.18). Hence, by (3.18) and (3.19), we see $Q_{0}\left[\chi\left(\cdot+2^{k}\right)\right](0) \leq 1 / 2$. So, from $\lim _{k \rightarrow \infty} \chi\left(\cdot+2^{k}\right)=1 / 2$ and Proposition 9, we obtain $Q_{0}[1 / 2] \leq 1 / 2$. This is a contradiction with Hypothesis 3 (iv).

Lemma 14. Let $Q^{t}$ be a map from $\mathcal{M}$ to $\mathcal{M}$ for $t \in[0,+\infty)$. Suppose $Q$ satisfies Hypothesis 4 (ii). Then, $\lim _{t \rightarrow 0}\left(Q^{t}[u]\right)(x-c t)=u(x)$ holds for all $c \in \mathbb{R}, u \in \mathcal{M}$ and continuous points $x \in \mathbb{R}$ of $u$.

Proof. Let a sequence $\left\{t_{k}\right\}_{k \in \mathbb{N}} \subset[0,+\infty)$ converge to 0 . Then, by Hypothesis 4 (ii) and Lemma 12, $\lim _{k \rightarrow \infty} Q^{t_{k}}[u]\left(x-c t_{k}\right)=u(x)$ holds for all continuous points $x \in \mathbb{R}$ of $u$.

Proof of Theorem 5. By Theorem 11, there exists $\psi_{k} \in \mathcal{M}$ with $Q^{\frac{\tau}{2^{k}}}\left[\psi_{k}\right]\left(\cdot-\frac{c \tau}{2^{k}}\right)=\psi_{k}(\cdot), \psi_{k}(-\infty)=0$ and $\psi_{k}(+\infty)=1$ for $k \in \mathbb{N}$. We also take $x_{k}$ such that $\psi_{k}\left(-x_{k}\right) \leq 1 / 2 \leq \lim _{h \downarrow+0} \psi_{k}\left(-x_{k}+h\right)$, and put $\psi^{k}(\cdot):=\psi_{k}\left(\cdot-x_{k}\right) \in \mathcal{M}$. Then, we have

$$
\psi^{k}(0) \leq 1 / 2 \leq \lim _{h \downarrow+0} \psi^{k}(h)
$$

and

$$
Q^{\frac{\tau}{2^{k}}}\left[\psi^{k}\right]\left(\cdot-\frac{c \tau}{2^{k}}\right)=\psi^{k}(\cdot) .
$$

By Helly's theorem, there exist a subsequence $\{k(n)\}_{n \in \mathbb{N}}$ and $\psi \in \mathcal{M}$ such that $\lim _{n \rightarrow \infty} \psi^{k(n)}(x)=\psi(x)$ holds for all continuous points $x \in \mathbb{R}$ of $\psi$.

Let $k_{0} \in \mathbb{N}$ and $m_{0} \in \mathbb{N}$. As $n \in \mathbb{N}$ is sufficiently large,

$$
Q^{\frac{m_{0} \tau}{2^{k_{0}}}}\left[\psi^{k(n)}\right]\left(\cdot-c \frac{m_{0} \tau}{2^{k_{0}}}\right)
$$




$$
=\left(Q^{\frac{\tau}{2^{k(n)}}}\right)^{m_{0} 2^{k(n)-k_{0}}}\left[\psi^{k(n)}\right]\left(\cdot-\frac{c \tau}{2^{k(n)}} m_{0} 2^{k(n)-k_{0}}\right)=\psi^{k(n)}(\cdot)
$$

holds because of $k(n) \geq k_{0}$ and (3.21). Therefore, by Proposition 9, we obtain

$$
Q^{\frac{m_{0} \tau}{2^{k_{0}}}}[\psi]\left(\cdot-c \frac{m_{0} \tau}{2^{k_{0}}}\right)=\psi(\cdot) .
$$

From (3.20), we also see

$$
\psi(0) \leq 1 / 2 \leq \lim _{h \downarrow+0} \psi(h) .
$$

Let $t \in[0,+\infty)$. Then, by $(3.22)$, there exists a sequence $\left\{t_{k}\right\}_{k \in \mathbb{N}} \subset$ $[0,+\infty)$ with $\lim _{k \rightarrow \infty} t_{k}=0$ such that $Q^{t+t_{k}}[\psi]\left(\cdot-c\left(t+t_{k}\right)\right)=\psi(\cdot)$ holds for all $k \in \mathbb{N}$. So, by $Q^{t_{k}}\left[Q^{t}[\psi](\cdot-c t)\right]\left(\cdot-c t_{k}\right)=Q^{t+t_{k}}[\psi]\left(\cdot-c\left(t+t_{k}\right)\right)$ and Lemma 14 , we obtain

$$
Q^{t}[\psi](\cdot-c t)=\psi(\cdot) .
$$

Hence, because $Q^{t}[\psi(-\infty)]=\psi(-\infty)$ and $Q^{t}[\psi(+\infty)]=\psi(+\infty)$ hold by Proposition 9, from (3.23), we see $\psi(-\infty)=0$ and $\psi(+\infty)=1$.

Proof of Theorem 6. In virtue of Theorem 13, we take $c_{*} \in(-\infty,+\infty]$ such that the following holds: Let $c \in \mathbb{R}$. Then, there exists $\phi \in \mathcal{M}$ with $\left(Q^{1}[\phi]\right)(\cdot-c) \equiv \phi(\cdot), \phi(-\infty)=0$ and $\phi(+\infty)=1$ if and only if $c \geq c_{*}$.

Then, from Theorem 5, we have the conclusion of this theorem.

\section{$\S 4$. Basic Facts for Nonlocal Equations in $L^{\infty}(\mathbb{R})$}

In this section, we give some basic facts for the equation

$$
u_{t}=\hat{\mu} * u+g(u)
$$

on the phase space $L^{\infty}(\mathbb{R})$. We do not necessarily assume $\hat{\mu}(\mathbb{R})=1$ or that the nonlinearity $\hat{\mu}(\mathbb{R}) u+g(u)$ is monostable. So, the equation (4.1) is more general than (1.1). This slight generalization would be useful to a sequel [30].

First, we have the comparison theorem for $(4.1)$ on $L^{\infty}(\mathbb{R})$ :

Lemma 15. Let $\hat{\mu}$ be a Borel-measure on $\mathbb{R}$ with $\hat{\mu}(\mathbb{R})<+\infty$. Let $g$ be a Lipschitz continuous function on $\mathbb{R}$. Let $T \in(0,+\infty)$, and two functions $u^{1}$ and $u^{2} \in C^{1}\left([0, T], L^{\infty}(\mathbb{R})\right)$. Suppose that for any $t \in[0, T]$, the inequality

$$
u_{t}^{1}-\left(\hat{\mu} * u^{1}+g\left(u^{1}\right)\right) \leq u_{t}^{2}-\left(\hat{\mu} * u^{2}+g\left(u^{2}\right)\right)
$$

holds almost everywhere in $x$. Then, the inequality $u^{1}(T, x) \leq u^{2}(T, x)$ holds almost everywhere in $x$ if the inequality $u^{1}(0, x) \leq u^{2}(0, x)$ holds almost everywhere in $x$. 
Proof. Put $K \in \mathbb{R}$ by

$$
K:=-\inf _{h>0, u \in \mathbb{R}} \frac{g(u+h)-g(u)}{h},
$$

and $v \in C^{1}\left([0, T], L^{\infty}(\mathbb{R})\right)$ by

$$
v(t):=e^{K t}\left(u^{2}-u^{1}\right)(t) .
$$

Then, we have the ordinary differential equation

$$
\frac{d v}{d t}=F(t, v)
$$

in $L^{\infty}(\mathbb{R})$ with $v(0)=\left(u^{2}-u^{1}\right)(0)$ as we define a map $F:[0, T] \times L^{\infty}(\mathbb{R}) \rightarrow$ $L^{\infty}(\mathbb{R})$ by

$$
F(t, w):=\hat{\mu} * w+K w+e^{K t}\left(g\left(u^{1}(t)+e^{-K t} w\right)-g\left(u^{1}(t)\right)\right)+e^{K t} a(t),
$$

where

$$
a:=\left(\frac{d u^{2}}{d t}-\left(\hat{\mu} * u^{2}+g\left(u^{2}\right)\right)\right)-\left(\frac{d u^{1}}{d t}-\left(\hat{\mu} * u^{1}+g\left(u^{1}\right)\right)\right) .
$$

For any $t \in[0, T]$, we see the inequality

$$
a(t, x) \geq 0
$$

almost everywhere in $x$. Take the solution $\tilde{v} \in C^{1}\left([0, T], L^{\infty}(\mathbb{R})\right)$ to

$$
\tilde{v}(t)=v(0)+\int_{0}^{t} \max \{F(s, \tilde{v}(s)), 0\} d s .
$$

Then, for any $t \in[0, T]$, we have

$$
\tilde{v}(t, x) \geq v(0, x)=\left(u^{2}-u^{1}\right)(0, x) \geq 0
$$

almost everywhere in $x$. By using (4.2), (4.5) and (4.7), for any $t \in[0, T]$, we also have the inequality $F(t, \tilde{v}(t)) \geq 0$ almost everywhere in $x$. Hence, from (4.6), $\tilde{v}(t)$ is the solution to the same ordinary differential equation (4.4) in $L^{\infty}(\mathbb{R})$ as $v(t)$ with $\tilde{v}(0)=v(0)$. So, in virtue of (4.3) and (4.7),

$$
\left(u^{2}-u^{1}\right)(T, x)=e^{-K T} v(T, x)=e^{-K T} \tilde{v}(T, x) \geq 0
$$

holds almost everywhere in $x$.

The following lemma gives a invariant set and some positively invariant sets of the flow on $L^{\infty}(\mathbb{R})$ generated by the equation (4.1): 
Lemma 16. Let $\hat{\mu}$ be a Borel-measure on $\mathbb{R}$ with $\hat{\mu}(\mathbb{R})<+\infty$. Let $g$ be a Lipschitz continuous function on $\mathbb{R}$. Then, the followings hold:

(i) For any $u_{0} \in B C(\mathbb{R})$, there exists a solution $\{u(t)\}_{t \in \mathbb{R}} \subset B C(\mathbb{R})$ to (4.1) with $u(0)=u_{0}$. Here, $B C(\mathbb{R})$ denote the set of bounded and continuous functions on $\mathbb{R}$.

(ii) Suppose a constant $\gamma$ satisfies $\gamma \hat{\mu}(\mathbb{R})+g(\gamma)=0$. If $u_{0} \in L^{\infty}(\mathbb{R})$ satisfies $\gamma \leq u_{0}$, then there exists a solution $\{u(t)\}_{t \in[0,+\infty)} \subset L^{\infty}(\mathbb{R})$ to (4.1) with $u(0)=u_{0}$ and $\gamma \leq u(t)$. If $u_{0} \in L^{\infty}(\mathbb{R})$ satisfies $u_{0} \leq \gamma$, then there exists a solution $\{u(t)\}_{t \in[0,+\infty)} \subset L^{\infty}(\mathbb{R})$ to (4.1) with $u(0)=u_{0}$ and $u(t) \leq \gamma$.

(iii) If $u_{0}$ is a bounded and monotone nondecreasing function on $\mathbb{R}$, then there exists a solution $\{u(t)\}_{t \in[0,+\infty)} \subset L^{\infty}(\mathbb{R})$ to $(4.1)$ with $u(0)=u_{0}$ such that $u(t)$ is a bounded and monotone nondecreasing function on $\mathbb{R}$ for all $t \in$ $[0,+\infty)$. If $u_{0}$ is a bounded and monotone nonincreasing function on $\mathbb{R}$, then there exists a solution $\{u(t)\}_{t \in[0,+\infty)} \subset L^{\infty}(\mathbb{R})$ to (4.1) with $u(0)=u_{0}$ such that $u(t)$ is a bounded and monotone nonincreasing function on $\mathbb{R}$ for all $t \in$ $[0,+\infty)$.

Proof. We could see (i), because $B C(\mathbb{R})$ is a closed sub-space of the Banach space $L^{\infty}(\mathbb{R})$ and $u \in B C(\mathbb{R})$ implies $\hat{\mu} * u+g(u) \in B C(\mathbb{R})$.

We could also see (ii) by using Lemma 15, because the constant $\gamma$ is a solution to (4.1).

We show (iii). Suppose $u_{0}$ is a bounded and monotone nondecreasing function on $\mathbb{R}$. We take a solution $\{u(t)\}_{t \in[0,+\infty)} \subset L^{\infty}(\mathbb{R})$ to (4.1) with $u(0)=u_{0}$. Let $t \in[0,+\infty)$ and $h \in[0,+\infty)$. Then, by Lemma 15, we see $u(t, x) \leq u(t, x+h)$ almost everywhere in $x$. We take a cutoff function $\rho \in C^{\infty}(\mathbb{R})$ with

$$
\begin{aligned}
& |x| \geq 1 / 2 \Longrightarrow \rho(x)=0, \\
& |x|<1 / 2 \Longrightarrow \rho(x)>0
\end{aligned}
$$

and

$$
\int_{x \in \mathbb{R}} \rho(x) d x=1
$$

As we put

$$
v_{n}(x):=\int_{y \in \mathbb{R}} 2^{n} \rho\left(2^{n}(x-y)\right) u(t, y) d y
$$

for $n \in \mathbb{N}$, we see $v_{n}(x) \leq v_{n}(x+h)$ for all $x \in \mathbb{R}$. Therefore, $v_{n}$ is smooth, bounded and monotone nondecreasing. By Helly's theorem, there exist a subsequence $n_{k}$ and a bounded and monotone nondecreasing function $\psi$ on $\mathbb{R}$ such that $\lim _{k \rightarrow \infty} v_{n_{k}}(x)=\psi(x)$ holds for all $x \in \mathbb{R}$. Then, $\| u(t, x)-$ 
$\psi(x) \|_{L^{1}([-C,+C])} \leq \lim _{k \rightarrow \infty}\left(\left\|u(t, x)-v_{n_{k}}(x)\right\|_{L^{1}([-C,+C])}+\right.$

$\left.\left\|v_{n_{k}}(x)-\psi(x)\right\|_{L^{1}([-C,+C])}\right)=0$ holds for all $C \in(0,+\infty)$. Hence, we obtain $\|u(t, x)-\psi(x)\|_{L^{\infty}(\mathbb{R})}=0$.

Lemma 17. Let $\hat{\mu}$ be a Borel-measure on $\mathbb{R}$ with $\hat{\mu}(\mathbb{R})<+\infty$. Let $\left\{u_{n}\right\}_{n=1}^{\infty}$ be a sequence of bounded and continuous functions on $\mathbb{R}$ with

$$
\sup _{n \in \mathbb{N}, x \in \mathbb{R}}\left|u_{n}(x)\right|<+\infty .
$$

Suppose the sequence $\left\{u_{n}\right\}_{n=1}^{\infty}$ converges to 0 uniformly on every bounded interval. Then, the sequence $\left\{\hat{\mu} * u_{n}\right\}_{n=1}^{\infty}$ converges to 0 uniformly on every bounded interval.

Proof. Let $\varepsilon \in(0,+\infty)$. We take a positive constant $C$ such that

$$
\left(\sup _{n \in \mathbb{N}, x \in \mathbb{R}}\left|u_{n}(x)\right|\right) \hat{\mu}(\mathbb{R} \backslash(-C,+C)) \leq \varepsilon
$$

holds. Then, because

$$
\begin{gathered}
\left|\left(\hat{\mu} * u_{n}\right)(x)\right| \leq \int_{y \in(-C,+C)}\left|u_{n}(x-y)\right| d \hat{\mu}(y)+\int_{y \in \mathbb{R} \backslash(-C,+C)}\left|u_{n}(x-y)\right| d \hat{\mu}(y) \\
\quad \leq\left(\sup _{y \in(-C,+C)}\left|u_{n}(x-y)\right|\right) \hat{\mu}(\mathbb{R})+\left(\sup _{y \in \mathbb{R}}\left|u_{n}(x-y)\right|\right) \hat{\mu}(\mathbb{R} \backslash(-C,+C))
\end{gathered}
$$

holds, we have

$$
\sup _{x \in[-I,+I]}\left|\left(\hat{\mu} * u_{n}\right)(x)\right| \leq\left(\sup _{y \in(-(I+C),+(I+C))}\left|u_{n}(y)\right|\right) \hat{\mu}(\mathbb{R})+\varepsilon
$$

for all $I \in(0,+\infty)$. Hence, we obtain

$$
\limsup _{n \rightarrow \infty} \sup _{x \in[-I,+I]}\left|\left(\hat{\mu} * u_{n}\right)(x)\right| \leq \varepsilon
$$

for all $I \in(0,+\infty)$.

Proposition 18. Let $\hat{\mu}$ be a Borel-measure on $\mathbb{R}$ with $\hat{\mu}(\mathbb{R})<+\infty, g$ a Lipschitz continuous function on $\mathbb{R}$, and $T$ a positive constant. Let a sequence $\left\{u_{n}\right\}_{n=0}^{\infty} \subset C^{1}\left([0, T], L^{\infty}(\mathbb{R})\right)$ of solutions to the equation (4.1) satisfy

$$
\sup _{n \in \mathbb{N}, x \in \mathbb{R}}\left|u_{n}(0, x)-u_{0}(0, x)\right|<+\infty .
$$


Suppose

$$
\lim _{n \rightarrow \infty} \sup _{x \in[-I,+I]}\left|u_{n}(0, x)-u_{0}(0, x)\right|=0
$$

holds for all positive constants I. Then,

$$
\lim _{n \rightarrow \infty} \sup _{t \in[0, T]}\left\|u_{n}(t, x)-u_{0}(t, x)\right\|_{L^{\infty}([-J,+J])}=0
$$

holds for all positive constants $J$.

Proof. First, we take a sequence $\left\{w_{n}\right\}_{n=1}^{\infty}$ of nonnegative, bounded and continuous functions on $\mathbb{R}$ with

$$
\sup _{n \in \mathbb{N}, x \in \mathbb{R}}\left|w_{n}(x)\right|<+\infty
$$

such that $\left\{w_{n}\right\}_{n=1}^{\infty}$ converges to 0 uniformly on every bounded interval and

$$
\left|u_{n}(0, x)-u_{0}(0, x)\right| \leq w_{n}(x)
$$

holds for all $n \in \mathbb{N}$ and $x \in \mathbb{R}$. Let $\hat{A}$ denote the bounded and linear operator from the Banach space $B C(\mathbb{R})$ to $B C(\mathbb{R})$ defined by

$$
\hat{A} w:=\hat{\mu} * w .
$$

From (4.8), we see $\sup _{n \in \mathbb{N}, x \in \mathbb{R}}\left|\left(\hat{A}^{k} w_{n}\right)(x)\right|<+\infty$ for all $k=0,1,2, \cdots$. Hence, because of $\lim _{n \rightarrow \infty} \sup _{x \in[-I,+I]}\left|w_{n}(x)\right|=0$ for all $I \in(0,+\infty)$, by Lemma 17, we have

$$
\lim _{n \rightarrow \infty} \sup _{x \in[-J,+J]}\left|\left(\hat{A}^{k} w_{n}\right)(x)\right|=0
$$

for all $J \in(0,+\infty)$ and $k=0,1,2, \cdots$.

Let $\gamma$ denote the constant defined by

$$
\gamma:=\sup _{h>0, u \in \mathbb{R}} \frac{g(u+h)-g(u)}{h} .
$$

Then, we consider the following two sequences $\left\{\underline{v}_{n}\right\}_{n=1}^{\infty}$ and $\left\{\bar{v}_{n}\right\}_{n=1}^{\infty} \subset C^{1}$ $\left([0, T], L^{\infty}(\mathbb{R})\right)$ defined by

$$
\underline{v}_{n}(t, x):=u_{0}(t, x)-e^{\gamma t}\left(e^{\hat{A} t} w_{n}\right)(x)
$$

and

$$
\bar{v}_{n}(t, x):=u_{0}(t, x)+e^{\gamma t}\left(e^{\hat{A} t} w_{n}\right)(x)
$$


Because $\left(e^{\hat{A} t} w_{n}\right)(x)$ is nonnegative for all $n \in \mathbb{N}, t \in[0,+\infty)$ and $x \in \mathbb{R}$, the function $\underline{v}_{n}$ is a sub-solution to (4.1) and $\bar{v}_{n}$ is a super-solution to (4.1) for all $n \in \mathbb{N}$. So, by Lemma 15 and (4.9), for any $n \in \mathbb{N}$ and $t \in[0, T]$,

$$
\left|u_{n}(t, x)-u_{0}(t, x)\right| \leq e^{\gamma t}\left(e^{\hat{A} t} w_{n}\right)(x)
$$

holds almost everywhere in $x$.

Let $\varepsilon \in(0,+\infty)$. We take $N \in \mathbb{N}$ such that

$$
\left(1+e^{\gamma T}\right)\left(\sum_{k=N}^{\infty} \frac{\left(T\|\hat{A}\|_{B C(\mathbb{R}) \rightarrow B C(\mathbb{R})}\right)^{k}}{k !}\right)\left(\sup _{n \in \mathbb{N}, x \in \mathbb{R}}\left|w_{n}(x)\right|\right) \leq \varepsilon
$$

holds. Then, in virtue of (4.11), we see

$$
\begin{gathered}
\left\|u_{n}(t, x)-u_{0}(t, x)\right\|_{L^{\infty}([-J,+J])} \leq \sup _{x \in[-J,+J]}\left|e^{\gamma t}\left(e^{\hat{A} t} w_{n}\right)(x)\right| \\
=e^{\gamma t}\left(\sup _{x \in[-J,+J]}\left|\left(\sum_{k=0}^{N-1} \frac{t^{k}}{k !}\left(\hat{A}^{k} w_{n}\right)(x)\right)+\left(\left(\sum_{k=N}^{\infty} \frac{t^{k}}{k !} \hat{A}^{k}\right) w_{n}\right)(x)\right|\right) \\
\leq\left(1+e^{\gamma T}\right)\left(\sum_{k=0}^{N-1} \frac{T^{k}}{k !}\left(\sup _{x \in[-J,+J]}\left|\left(\hat{A}^{k} w_{n}\right)(x)\right|\right)\right)+\varepsilon
\end{gathered}
$$

for all $J \in(0,+\infty), n \in \mathbb{N}$ and $t \in[0, T]$. So, by (4.10), we obtain

$$
\limsup _{n \rightarrow \infty} \sup _{t \in[0, T]}\left\|u_{n}(t, x)-u_{0}(t, x)\right\|_{L^{\infty}([-J,+J])} \leq \varepsilon
$$

for all $J \in(0,+\infty)$.

\section{$\S 5 . \quad$ Proof of Theorem 1}

In this section, we prove Theorem 1. The argument in this section is almost similar as in [29]. First, we recall that $\mu$ is a Borel-measure on $\mathbb{R}$ with $\mu(\mathbb{R})=1, f$ is a Lipschitz continuous function on $\mathbb{R}$ with $f(0)=f(1)=0$ and $f>0$ in $(0,1)$ and the set $\mathcal{M}$ has been defined at the beginning of Section 2 . Then, in virtue of Lemmas 15, 16 and Proposition 18, $Q^{t}(t \in(0,+\infty))$ satisfies Hypotheses 3 and $Q$ Hypotheses 4 for the semiflow $Q=\left\{Q^{t}\right\}_{t \in[0,+\infty)}$ on $\mathcal{M}$ generated by (1.1). So, Theorems 5 and 6 can work for this semiflow on $\mathcal{M}$.

If the flow on $L^{\infty}(\mathbb{R})$ generated by (1.1) has a periodic traveling wave solution with average speed $c$ (even if the profile is not a monotone function), then it has a traveling wave solution with monotone profile and speed $c$ : 
Theorem 19. Let $c \in \mathbb{R}$. Suppose there exist a positive constant $\tau$ and a solution $\{u(t, x)\}_{t \in \mathbb{R}} \subset L^{\infty}(\mathbb{R})$ to (1.1) with $0 \leq u(t, x) \leq 1, \lim _{x \rightarrow+\infty} u(t$, $x)=1$ and $\|u(t, x)-1\|_{L^{\infty}(\mathbb{R})} \neq 0$ such that

$$
u(t+\tau, x)=u(t, x+c \tau)
$$

holds for all $t$ and $x \in \mathbb{R}$. Then, there exists $\psi \in \mathcal{M}$ with $\psi(-\infty)=0$ and $\psi(+\infty)=1$ such that $\{\psi(x+c t)\}_{t \in \mathbb{R}}$ is a solution to (1.1).

Proof. Put two monotone nondecreasing functions $\varphi(x):=\max \{\alpha \in$ $\mathbb{R} \mid \alpha \leq u(0, y)$ holds almost everywhere in $y \in(x,+\infty)\}$ and $\phi(x):=\lim _{h \downarrow+0}$ $\varphi(x-h)$. Then, $\phi \in \mathcal{M}, \phi(-\infty)<1$ and $\phi(+\infty)=1$ hold. We take a cutoff function $\rho \in C^{\infty}(\mathbb{R})$ with

$$
\begin{aligned}
& |x+1 / 2| \geq 1 / 2 \Longrightarrow \rho(x)=0, \\
& |x+1 / 2|<1 / 2 \Longrightarrow \rho(x)>0
\end{aligned}
$$

and

$$
\int_{x \in \mathbb{R}} \rho(x) d x=1
$$

As we put

$$
v_{n}(x):=\int_{y \in \mathbb{R}} 2^{n} \rho\left(2^{n}(x-y)\right) u(0, y) d y
$$

for $n \in \mathbb{N}$, we see $\phi \leq v_{n}$. Let $N \in \mathbb{N}$. Because of $\lim _{n \rightarrow \infty} \| v_{n}(x)-$ $u(0, x) \|_{L^{1}([-N,+N])}=0$, there exists a subsequence $n_{k}$ such that $\lim _{k \rightarrow \infty} v_{n_{k}}(x)$ $=u(0, x)$ almost everywhere in $x \in[-N,+N]$. Therefore, we have $\phi(x) \leq$ $u(0, x)$ almost everywhere in $x \in \mathbb{R}$. So, by Lemma 15 , we obtain $Q^{\tau}[\phi](x-$ $c \tau) \leq u(\tau, x-c \tau)=u(0, x)$ almost everywhere in $x$. Hence, because $Q^{\tau}[\phi]$ $(x-c \tau) \leq \max \left\{\alpha \in \mathbb{R} \mid \alpha \leq Q^{\tau}[\phi](y-c \tau)\right.$ holds almost everywhere in $y \in$ $(x,+\infty)\} \leq \varphi(x)$ holds, we get $Q^{\tau}[\phi](x-c \tau)=\lim _{h \downarrow+0} Q^{\tau}[\phi]((x-h)-c \tau) \leq$ $\phi(x)$. Therefore, by Theorem 5 , there exists $\psi \in \mathcal{M}$ with $\psi(-\infty)=0$ and $\psi(+\infty)=1$ such that $Q^{t}[\psi](x-c t) \equiv \psi(x)$ holds for all $t \in[0,+\infty)$.

The infimum $c_{*}$ of the speeds of traveling wave solutions is not $-\infty$, and there is a traveling wave solution with speed $c$ when $c \geq c_{*}$ :

Lemma 20. There exists $c_{*} \in(-\infty,+\infty]$ such that the following holds:

Let $c \in \mathbb{R}$. Then, there exists $\psi \in \mathcal{M}$ with $\psi(-\infty)=0$ and $\psi(+\infty)=1$ such that $\{\psi(x+c t)\}_{t \in \mathbb{R}}$ is a solution to (1.1) if and only if $c \geq c_{*}$. 
Proof. It follows from Theorem 6 .

Proof of Theorem 1. Let $c_{*}$ denote the infimum of the speeds of traveling wave solutions with monotone profile. Then, in virtue of Theorem 19 and Lemma 20, it is sufficient if we show $c_{*} \neq+\infty$.

Take $K \in[0,+\infty)$ such that

$$
K \geq \max \left\{\int_{y \in \mathbb{R}} e^{-\lambda y} d \mu(y), \mu(\mathbb{R})\right\}-1+\sup _{h>0} \frac{f(h)}{h} .
$$

As we put $\phi(x):=\min \left\{e^{\lambda x}, 1\right\} \in \mathcal{M}$, we see

$$
\begin{gathered}
(\mu * \phi)(x) \leq \min \left\{\left(\int_{y \in \mathbb{R}} e^{-\lambda y} d \mu(y)\right) e^{\lambda x}, \mu(\mathbb{R})\right\} \\
\leq \max \left\{\int_{y \in \mathbb{R}} e^{-\lambda y} d \mu(y), \mu(\mathbb{R})\right\} \phi(x) .
\end{gathered}
$$

So, $e^{K t} \phi(x)$ is a super-solution to (1.1), because of

$$
e^{K t}(\mu * \phi)-e^{K t} \phi+f\left(e^{K t} \phi\right) \leq K e^{K t} \phi .
$$

Hence, by Lemma 15, we obtain $Q^{1}[\phi](x) \leq e^{K} \phi(x) \leq e^{\lambda\left(x+\frac{K}{\lambda}\right)}$, and $Q^{1}[\phi](x-$ $\left.\frac{K}{\lambda}\right) \leq \phi(x)$. Therefore, from Theorem 5 , there exists $\psi \in \mathcal{M}$ with $\psi(-\infty)=0$ and $\psi(+\infty)=1$ such that $Q^{t}[\psi]\left(x-\frac{K}{\lambda} t\right) \equiv \psi(x)$ holds for all $t \in[0,+\infty)$. So, $c_{*} \leq \frac{K}{\lambda}$ holds.

\section{§6. A Result on Spreading Speeds by Weinberger}

In this section, we recall a result by Weinberger [25]. In Section 7 below, we use it to prove Theorem 2. Put a set of functions on $\mathbb{R}$;

$$
\mathcal{B}:=\{u \mid u \text { is a continuous function on } \mathbb{R} \text { with } 0 \leq u \leq 1\} .
$$

Hypotheses 21. Let $\tilde{Q}_{0}$ be a map from $\mathcal{B}$ into $\mathcal{B}$.

(i) $\tilde{Q}_{0}$ is continuous in the following sense: If a sequence $\left\{u_{k}\right\}_{k \in \mathbb{N}} \subset \mathcal{B}$ converges to $u \in \mathcal{B}$ uniformly on every bounded interval, then the sequence $\left\{\left(\tilde{Q}_{0}\left[u_{k}\right]\right)(x)\right\}_{k \in \mathbb{N}}$ converges to $\left(\tilde{Q}_{0}[u]\right)(x)$ for all $x \in \mathbb{R}$.

(ii) $\tilde{Q}_{0}$ is order preserving; i.e.,

$$
u_{1} \leq u_{2} \Longrightarrow \tilde{Q}_{0}\left[u_{1}\right] \leq \tilde{Q}_{0}\left[u_{2}\right]
$$

for all $u_{1}$ and $u_{2} \in \mathcal{B}$. Here, $u \leq v$ means that $u(x) \leq v(x)$ holds for all $x \in \mathbb{R}$. 
(iii) $\tilde{Q}_{0}$ is translation invariant; i.e.,

$$
T_{x_{0}} \tilde{Q}_{0}=\tilde{Q}_{0} T_{x_{0}}
$$

for all $x_{0} \in \mathbb{R}$. Here, $T_{x_{0}}$ is the translation operator defined by $\left(T_{x_{0}}[u]\right)(\cdot):=$ $u\left(\cdot-x_{0}\right)$.

(iv) $\tilde{Q}_{0}$ is monostable; i.e.,

$$
0<\gamma<1 \Longrightarrow \gamma<\tilde{Q}_{0}[\gamma]
$$

for all constant functions $\gamma$, and $\tilde{Q}_{0}[0]=0$.

Remark. If $\tilde{Q}_{0}$ satisfies Hypotheses 21 (ii) and (iii), then $\tilde{Q}_{0}$ maps monotone functions to monotone functions.

Theorem 22. $\quad$ Let a map $\tilde{Q}_{0}: \mathcal{B} \rightarrow \mathcal{B}$ satisfy Hypotheses 21. Let a continuous and monotone nonincreasing function $\varphi$ on $\mathbb{R}$ with $0<\varphi(-\infty)<1$ satisfy $\varphi(x)=0$ for all $x \in[0,+\infty)$. For $c \in \mathbb{R}$, define the sequence $\left\{a_{c, n}\right\}_{n=0}^{\infty}$ of continuous and monotone nonincreasing functions on $\mathbb{R}$ by the recursion

$$
a_{c, n+1}(x):=\max \left\{\left(\tilde{Q}_{0}\left[a_{c, n}\right]\right)(x+c), \varphi(x)\right\}
$$

with $a_{c, 0}:=\varphi$. Then, the inequality

$$
0 \leq a_{c, n} \leq a_{c, n+1} \leq 1
$$

holds for all $c \in \mathbb{R}$ and $n=0,1,2, \cdots$. For $c \in \mathbb{R}$, define the bounded and monotone nonincreasing function $a_{c}$ on $\mathbb{R}$ by

$$
a_{c}(x):=\lim _{n \rightarrow \infty} a_{c, n}(x)
$$

Let $\tilde{\nu}$ be a Borel-measure on $\mathbb{R}$ with $1<\tilde{\nu}(\mathbb{R})<+\infty$. Suppose there exists a positive constant $\varepsilon$ such that the inequality

$$
\tilde{\nu} * u \leq \tilde{Q}_{0}[u]
$$

holds for all $u \in \mathcal{B}$ with $u \leq \varepsilon$. Then, the inequality

$$
\inf _{\lambda>0} \frac{1}{\lambda} \log \int_{y \in \mathbb{R}} e^{\lambda y} d \tilde{\nu}(y) \leq \sup \left\{c \in \mathbb{R} \mid a_{c}(+\infty)=1\right\}
$$

holds. 
Proof. It follows from Lemma 5.4 and Theorem 6.4 in [25] with $N:=1$, $\mathcal{H}:=\mathbb{R}, \pi_{0}:=0, \pi_{1}=\pi_{+}:=1, S^{N-1}:=\{ \pm 1\}$ and $\xi:=+1$.

From Theorem 22, we have the following:

Proposition 23. Let $\hat{\mu}$ be a Borel-measure on $\mathbb{R}$ with $\hat{\mu}(\mathbb{R})=1$. Let $c_{0} \in \mathbb{R}$, and $\hat{\psi}$ be a monotone nonincreasing function on $\mathbb{R}$ with $\hat{\psi}(-\infty)=1$ and $\hat{\psi}(+\infty)=0$. Suppose $\left\{\hat{\psi}\left(x-c_{0} t\right)\right\}_{t \in \mathbb{R}} \subset L^{\infty}(\mathbb{R})$ is a solution to

$$
u_{t}=\hat{\mu} * u-u+f(u)
$$

Let $\tilde{Q}_{0}: \mathcal{B} \rightarrow \mathcal{B}$ be the time 1 map of the semiflow on $\mathcal{B}$ generated by the equation (6.1). Let $\tilde{\nu}$ be a Borel-measure on $\mathbb{R}$ with $1<\tilde{\nu}(\mathbb{R})<+\infty$. Suppose there exists a positive constant $\varepsilon$ such that the inequality

$$
\tilde{\nu} * u \leq \tilde{Q}_{0}[u]
$$

holds for all $u \in \mathcal{B}$ with $u \leq \varepsilon$. Then, the inequality

$$
\inf _{\lambda>0} \frac{1}{\lambda} \log \int_{y \in \mathbb{R}} e^{\lambda y} d \tilde{\nu}(y) \leq c_{0}
$$

holds.

Proof. We take a continuous and monotone nonincreasing function $\varphi$ on $\mathbb{R}$ with $0<\varphi(-\infty)<1$ and $\varphi(x)=0$ for all $x \in[0,+\infty)$. For $c \in \mathbb{R}$, we define the sequence $\left\{a_{c, n}\right\}_{n=0}^{\infty}$ of continuous and monotone nonincreasing functions on $\mathbb{R}$ by the recursion

$$
a_{c, n+1}(x):=\max \left\{\left(\tilde{Q}_{0}\left[a_{c, n}\right]\right)(x+c), \varphi(x)\right\}
$$

with $a_{c, 0}:=\varphi$. We also take $x_{0} \in \mathbb{R}$ such that

$$
\varphi(x) \leq \hat{\psi}\left(x-x_{0}\right)
$$

holds for all $x \in \mathbb{R}$.

Let $c \in\left[c_{0},+\infty\right)$. Then, we show $a_{c, n}(x) \leq \hat{\psi}\left(x-x_{0}\right)$ for all $n=0,1,2, \cdots$. We have $a_{c, 0}(x)=\varphi(x) \leq \hat{\psi}_{0}\left(x-x_{0}\right)$. As $a_{c, n}(x) \leq \hat{\psi}\left(x-x_{0}\right)$ holds almost everywhere in $x$,

$$
\begin{gathered}
a_{c, n+1}(x) \leq \max \left\{\left(\tilde{Q}_{0}\left[a_{c, n}\right]\right)\left(x+c_{0}\right), \varphi(x)\right\} \\
\leq \max \left\{\hat{\psi}\left(x-x_{0}\right), \varphi(x)\right\}=\hat{\psi}\left(x-x_{0}\right)
\end{gathered}
$$


also holds almost everywhere in $x$, because $\hat{\psi}\left(x-x_{0}-c_{0} t\right)$ is a solution to (6.1) and so $\left(\tilde{Q}_{0}\left[a_{c, n}\right]\right)(x) \leq \hat{\psi}\left(x-x_{0}-c_{0}\right)$ holds almost everywhere in $x$. So, for any $n=0,1,2, \cdots$, the inequality $a_{c, n}(x) \leq \hat{\psi}\left(x-x_{0}\right)$ holds almost everywhere in $x$. Hence, because $a_{c, n}$ is continuous and $\hat{\psi}$ is monotone, we have

$$
a_{c, n}(x) \leq \hat{\psi}\left(x-x_{0}\right)
$$

for all $x \in \mathbb{R}, c \in\left[c_{0},+\infty\right)$ and $n=0,1,2, \cdots$. Therefore, by Theorem 22, (6.2) and $\hat{\psi}(+\infty)=0$, the inequality

$$
\inf _{\lambda>0} \frac{1}{\lambda} \log \int_{y \in \mathbb{R}} e^{\lambda y} d \tilde{\nu}(y) \leq \sup \left(\mathbb{R} \backslash\left[c_{0},+\infty\right)\right)=c_{0}
$$

holds.

\section{$\S 7 . \quad$ Proof of Theorem 2}

In this section, we prove Theorem 2. First, we give a basic fact for the linear equation

$$
v_{t}=\hat{\mu} * v
$$

on the phase space $B C(\mathbb{R})$ :

Lemma 24. Let $\hat{\mu}$ be a Borel-measure on $\mathbb{R}$ with $\hat{\mu}(\mathbb{R})<+\infty$. Let $\hat{P}: B C(\mathbb{R}) \rightarrow B C(\mathbb{R})$ be the time 1 map of the flow on $B C(\mathbb{R})$ generated by the linear equation (7.1). Then, there exists a Borel-measure $\hat{\nu}$ on $\mathbb{R}$ with $\hat{\nu}(\mathbb{R})<+\infty$ such that

$$
\hat{P}[v]=\hat{\nu} * v
$$

holds for all $v \in B C(\mathbb{R})$. Further, if $v$ is a nonnegative, bounded and continuous function on $\mathbb{R}$, then the inequality

$$
v+\hat{\mu} * v \leq \hat{\nu} * v
$$

holds.

Proof. Put a functional $\tilde{P}: B C(\mathbb{R}) \rightarrow \mathbb{R}$ as

$$
\tilde{P}[v]:=(\hat{P}[v])(0) .
$$

Then, the functional $\tilde{P}$ is linear, bounded and positive. Hence, there exists a Borel-measure $\tilde{\nu}$ on $\mathbb{R}$ with $\tilde{\nu}(\mathbb{R})<+\infty$ such that if a continuous function $v$ on $\mathbb{R}$ satisfies $\lim _{|x| \rightarrow \infty} v(x)=0$, then

$$
\tilde{P}[v]=\int_{y \in \mathbb{R}} v(y) d \tilde{\nu}(y)
$$


holds.

Let $v \in B C(\mathbb{R})$. Then, there exists a sequence $\left\{v_{n}\right\}_{n=1}^{\infty} \subset B C(\mathbb{R})$ with $\sup _{n \in \mathbb{N}, x \in \mathbb{R}}\left|v_{n}(x)\right|<+\infty$ and $\lim _{|x| \rightarrow \infty} v_{n}(x)=0$ for all $n \in \mathbb{N}$ such that $v_{n} \rightarrow v$ as $n \rightarrow \infty$ uniformly on every bounded interval. From Proposition 18, $(7.2)$ and $\tilde{\nu}(\mathbb{R})<+\infty$, we have

$$
\tilde{P}[v]=\lim _{n \rightarrow \infty} \tilde{P}\left[v_{n}\right]=\lim _{n \rightarrow \infty} \int_{y \in \mathbb{R}} v_{n}(y) d \tilde{\nu}(y)=\int_{y \in \mathbb{R}} v(y) d \tilde{\nu}(y) .
$$

We take a Borel-measure $\hat{\nu}$ on $\mathbb{R}$ with $\hat{\nu}(\mathbb{R})<+\infty$ such that

$$
\hat{\nu}((-\infty, y))=\tilde{\nu}((-y,+\infty))
$$

holds for all $y \in \mathbb{R}$. Then, for any $v \in B C(\mathbb{R})$, we have

$$
(\hat{P}[v])(x) \equiv \tilde{P}[v(\cdot+x)] \equiv \int_{y \in \mathbb{R}} v(y+x) d \tilde{\nu}(y) \equiv(\hat{\nu} * v)(x) .
$$

Let $v$ be a nonnegative, bounded and continuous function on $\mathbb{R}$. Then, in $t \in[0,+\infty)$, the function

$$
u(t, x):=v(x)+t(\hat{\mu} * v)(x)
$$

is a sub-solution to (7.1), because of $v(x) \leq u(t, x)$. Hence,

$$
v+\hat{\mu} * v \leq \hat{P}[v]
$$

holds.

Lemma 25. Let $\hat{\mu}$ be a Borel-measure on $\mathbb{R}$ with $\hat{\mu}(\mathbb{R})<+\infty$. Suppose a constant $\gamma$ and a Lipschitz continuous function $g$ on $\mathbb{R}$ with $g(0)=0$ satisfy $\gamma<g^{\prime}(0)$. Let $\tilde{P}: B C(\mathbb{R}) \rightarrow B C(\mathbb{R})$ be the time 1 map of the flow on $B C(\mathbb{R})$ generated by the linear equation

$$
v_{t}=\hat{\mu} * v+\gamma v \text {. }
$$

Let $\tilde{P}_{0}: B C(\mathbb{R}) \rightarrow B C(\mathbb{R})$ be the time 1 map of the flow on $B C(\mathbb{R})$ generated by the equation

$$
v_{t}=\hat{\mu} * v+g(v) .
$$

Then, there exists a positive constant $\varepsilon$ such that the inequality

$$
\tilde{P}[v] \leq \tilde{P}_{0}[v]
$$

holds for all $v \in B C(\mathbb{R})$ with $0 \leq v \leq \varepsilon$. 
Proof. We take a positive constant $\varepsilon$ such that

$$
h \in\left[0,\left(1+e^{\hat{\mu}(\mathbb{R})+\gamma}\right) \varepsilon\right] \Longrightarrow \gamma h \leq g(h)
$$

holds. Let a function $v \in B C(\mathbb{R})$ satisfy $0 \leq v \leq \varepsilon$. Then, we take the solution $\tilde{v}(t, x)$ to $(7.3)$ with $\tilde{v}(0, x)=v(x)$. We see

$$
0 \leq \tilde{v}(t, x) \leq e^{(\hat{\mu}(\mathbb{R})+\gamma) t} \varepsilon \leq\left(1+e^{\hat{\mu}(\mathbb{R})+\gamma}\right) \varepsilon
$$

for all $t \in[0,1]$. Hence, from (7.5), in $t \in[0,1]$, the function $\tilde{v}(t, x)$ is a sub-solution to (7.4). So, the inequality

$$
(\tilde{P}[v])(x)=\tilde{v}(1, x) \leq\left(\tilde{P}_{0}[v]\right)(x)
$$

holds.

We use Proposition 23, Lemmas 24 and 25 to show the following:

Lemma 26. Let $f^{\prime}(0)>0$. Suppose there exist $c \in \mathbb{R}$ and $\psi \in \mathcal{M}$ with $\psi(-\infty)=0$ and $\psi(+\infty)=1$ such that $\{\psi(x+c t)\}_{t \in \mathbb{R}}$ is a solution to (1.1). Then, there exists a positive constant $\lambda$ such that

$$
\int_{y \in \mathbb{R}} e^{-\lambda y} d \mu(y)<+\infty
$$

holds.

Proof. Let $\hat{\mu}$ be the Borel-measure on $\mathbb{R}$ with $\hat{\mu}(\mathbb{R})=1$ such that

$$
\hat{\mu}((-\infty, y))=\mu((-y,+\infty))
$$

holds for all $y \in \mathbb{R}$. Let $\hat{P}: B C(\mathbb{R}) \rightarrow B C(\mathbb{R})$ be the time 1 map of the flow on $B C(\mathbb{R})$ generated by the linear equation (7.1). Then, by Lemma 24 , there exists a Borel-measure $\hat{\nu}$ on $\mathbb{R}$ with $\hat{\nu}(\mathbb{R})<+\infty$ such that for any $v \in B C(\mathbb{R})$,

$$
\hat{P}[v]=\hat{\nu} * v
$$

holds and for any nonnegative, bounded and continuous function $v$ on $\mathbb{R}$,

$$
\hat{\mu} * v \leq \hat{\nu} * v
$$

holds. Let $\tilde{P}: B C(\mathbb{R}) \rightarrow B C(\mathbb{R})$ be the time 1 map of the flow on $B C(\mathbb{R})$ generated by the linear equation

$$
v_{t}=\hat{\mu} * v-v+\frac{f^{\prime}(0)}{2} v .
$$


Then, from (7.6) and (7.7), as $\tilde{\nu}$ is the Borel-measure on $\mathbb{R}$ defined by

$$
\tilde{\nu}:=e^{-1+\frac{f^{\prime}(0)}{2}} \hat{\nu}
$$

we have

$$
\tilde{P}[v]=\tilde{\nu} * v
$$

for all $v \in B C(\mathbb{R})$ and

$$
\hat{\mu} * v \leq e^{1-\frac{f^{\prime}(0)}{2}}(\tilde{\nu} * v)
$$

for all nonnegative, bounded and continuous functions $v$ on $\mathbb{R}$. Because $\tilde{\nu}(\mathbb{R})=$ $(\tilde{\nu} * 1)(0)=(\tilde{P}[1])(0)=e^{\frac{f^{\prime}(0)}{2}}$ holds from $(7.8)$, we also have

$$
1<\tilde{\nu}(\mathbb{R})<+\infty
$$

Let $\tilde{Q}_{0}: \mathcal{B} \rightarrow \mathcal{B}$ be the time 1 map of the semiflow on $\mathcal{B}$ generated by the equation (6.1). Then, from Lemma 25 and (7.8), there exists a positive constant $\varepsilon$ such that the inequality

$$
\tilde{\nu} * u=\tilde{P}[u] \leq \tilde{Q}_{0}[u]
$$

holds for all $u \in \mathcal{B}$ with $u \leq \varepsilon$. Further, $\hat{\psi}(x-c t):=\psi(-(x-c t))$ is a solution to (6.1). Therefore, by Proposition 23 and (7.10), we obtain the inequality

$$
\inf _{\lambda>0} \frac{1}{\lambda} \log \int_{y \in \mathbb{R}} e^{\lambda y} d \tilde{\nu}(y) \leq c .
$$

So, there exists a positive constant $\lambda$ such that

$$
\int_{y \in \mathbb{R}} e^{\lambda y} d \tilde{\nu}(y) \leq e^{\lambda(c+1)}<+\infty
$$

holds. Hence, from (7.9), the inequality

$$
\begin{aligned}
& \int_{y \in \mathbb{R}} e^{-\lambda y} d \mu(y)=\int_{y \in \mathbb{R}} e^{\lambda y} d \hat{\mu}(y)=\lim _{n \rightarrow \infty} \int_{y \in \mathbb{R}} \min \left\{e^{\lambda y}, n\right\} d \hat{\mu}(y) \\
= & \lim _{n \rightarrow \infty}\left(\hat{\mu} * \min \left\{e^{-\lambda x}, n\right\}\right)(0) \leq e^{1-\frac{f^{\prime}(0)}{2}} \lim _{n \rightarrow \infty}\left(\tilde{\nu} * \min \left\{e^{-\lambda x}, n\right\}\right)(0) \\
= & e^{1-\frac{f^{\prime}(0)}{2}} \lim _{n \rightarrow \infty} \int_{y \in \mathbb{R}} \min \left\{e^{\lambda y}, n\right\} d \tilde{\nu}(y)=e^{1-\frac{f^{\prime}(0)}{2}} \int_{y \in \mathbb{R}} e^{\lambda y} d \tilde{\nu}(y)<+\infty
\end{aligned}
$$
holds.

Proof of Theorem 2. It follows from Theorem 19 and Lemma 26. 


\section{Acknowledgments}

I thank Prof. Hiroshi Matano, Dr. Xiaotao Lin and Dr. Masahiko Shimojo for their discussion. It was partially supported by Grant-in-Aid for Scientific Research (No.19740092) from Ministry of Education, Culture, Sports, Science and Technology, Japan.

\section{References}

[1] C. Atkinson and G. E. H. Reuter, Deterministic epidemic waves, Math. Proc. Cambridge Philos. Soc. 80 (1976), no. 2, 315-330.

[2] P. W. Bates, P. C. Fife, X. Ren and X. Wang, Traveling waves in a convolution model for phase transitions, Arch. Rational Mech. Anal. 138 (1997), no. 2, 105-136.

[3] J. Carr and A. Chmaj, Uniqueness of travelling waves for nonlocal monostable equations, Proc. Amer. Math. Soc. 132 (2004), no. 8, 2433-2439 (electronic).

[4] X. Chen, Existence, uniqueness, and asymptotic stability of traveling waves in nonlocal evolution equations, Adv. Differential Equations 2 (1997), no. 1, 125-160.

[5] X. Chen and J.-S. Guo, Uniqueness and existence of traveling waves for discrete quasilinear monostable dynamics, Math. Ann. 326 (2003), no. 1, 123-146.

[6] J. Coville, J. Dávila and S. Martínez, Nonlocal anisotropic dispersal with monostable nonlinearity, J. Differential Equations 244 (2008), no. 12, 3080-3118.

[7] J. Coville and L. Dupaigne, On a non-local equation arising in population dynamics, Proc. Roy. Soc. Edinburgh Sect. A 137 (2007), no. 4, 727-755.

[8] R. A. Fisher, The genetical theory of natural selection, A complete variorum edition, Revised reprint of the 1930 original, Oxford Univ. Press, Oxford, 1999.

[9] _ The wave of advance of advantageous genes, Ann. Eugenics 7 (1937), 335-369.

[10] B. H. Gilding and R. Kersner, Travelling waves in nonlinear diffusion-convection reaction, Birkhäuser, Basel, 2004.

[11] J.-S. Guo and F. Hamel, Front propagation for discrete periodic monostable equations, Math. Ann. 335 (2006), no. 3, 489-525.

[12] J.-S. Guo and Y. Morita, Entire solutions of reaction-diffusion equations and an application to discrete diffusive equations, Discrete Contin. Dyn. Syst. 12 (2005), no. 2, 193-212.

[13] F. Hamel and N. Nadirashvili, Entire solutions of the KPP equation, Comm. Pure Appl. Math. 52 (1999), no. 10, 1255-1276.

[14] Y. Hosono, The minimal speed for a diffusive Lotka-Volterra model, Bull. Math. Biol. 60 (1998), 435-448.

[15] Y. Kan-on, Fisher wave fronts for the Lotka-Volterra competition model with diffusion, Nonlinear Anal. 28 (1997), no. 1, 145-164.

[16] A. N. Kolmogorov, I. G. Petrovsky and N. S. Piskunov, Étude de l'équation de la difusion avec croissance de la quantité de matière et son application à un problème biologique, Bull. Univ. Moskov. Ser. Internat. A 1 (1937), 1-25.

[17] B. Li, H. F. Weinberger and M. A. Lewis, Spreading speeds as slowest wave speeds for cooperative systems, Math. Biosci. 196 (2005), no. 1, 82-98.

[18] X. Liang, Y. Yi and X.-Q. Zhao, Spreading speeds and traveling waves for periodic evolution systems, J. Differential Equations 231 (2006), no. 1, 57-77.

[19] X. Liang and X.-Q. Zhao, Asymptotic speeds of spread and traveling waves for monotone semiflows with applications, Comm. Pure Appl. Math. 60 (2007), no. 1, 1-40.

[20] H. Okamoto and M. Shōji, The mathematical theory of permanent progressive waterwaves, World Sci. Publishing, River Edge, NJ, 2001. 
[21] K. Schumacher, Travelling-front solutions for integro-differential equations. I, J. Reine Angew. Math. 316 (1980), 54-70.

[22] _ Travelling-front solutions for integro-differential equations. II, in Biological growth and spread (Proc. Conf., Heidelberg, 1979), 296-309, Lecture Notes in Biomath., 38, Springer, Berlin, 1980.

[23] K. Uchiyama, The behavior of solutions of some nonlinear diffusion equations for large time, J. Math. Kyoto Univ. 18 (1978), no. 3, 453-508.

[24] A. I. Volpert, Vitaly A. Volpert and Vladimir A. Volpert, Traveling wave solutions of parabolic systems, Translated from the Russian manuscript by James F. Heyda, Amer. Math. Soc., Providence, RI, 1994.

[25] H. F. Weinberger, Long-time behavior of a class of biological models, SIAM J. Math. Anal. 13 (1982), no. 3, 353-396.

[26] On spreading speeds and traveling waves for growth and migration models in a periodic habitat, J. Math. Biol. 45 (2002), no. 6, 511-548.

[27] H. F. Weinberger, M. A. Lewis and B. Li, Analysis of linear determinacy for spread in cooperative models, J. Math. Biol. 45 (2002), no. 3, 183-218.

[28] , Anomalous spreading speeds of cooperative recursion systems, J. Math. Biol. 55 (2007), no. 2, 207-222.

[29] H. Yagisita, Existence of traveling waves for a nonlocal monostable equation: an abstract approach, preprint (http://arxiv.org/pdf/0807.3612).

[30] _ Existence of traveling wave solutions for a nonlocal bistable equation: an abstract approach, Publ. Res. Inst. Math. Sci., submitted. 